\title{
Validación de contenido de un cuestionario de satisfacción usuaria con la atención abierta en un policlínico ambulatorio de otorrinolaringología
}

\section{User satisfaction with open attention on an outpatient otolaryngology clinic}

\author{
Felipe Cardemil M1,2, Daniel Muñoz S³ Álvaro Galindo C³ , Maritza Rahal E1.
}

\begin{abstract}
RESUMEN
Introducción: La satisfacción de los pacientes con respecto a la atención en salud que ofrecen las instituciones es relevante.

Objetivo: Realizar validación de contenido de un cuestionario que permita analizar la opinión de un grupo de pacientes consultantes a un policlínico de otorrinolaringología respecto al proceso de atención médica.

Material y método: Estudio de corte transversal. Se validó un instrumento mediante el método de Lawshe. Se crearon 7 reactivos, con un total de 83 preguntas para ser sometidas a análisis.

Resultados: Se validaron 25 preguntas que conformaron el cuestionario final, con una razón de validez de contenido de 0,75 o más. Fueron encuestados 120 pacientes entre 30 y 60 años, correspondientes a 83 mujeres (69,1\%). Del total, 75 pacientes (62,5\%) acudían por primera vez a consulta, y 36 pacientes (30\%) habían sido operados. Más del $94 \%$ de las pacientes refirió que el médico les explicó claramente su diagnóstico y dio con claridad las indicaciones para tratar su enfermedad. El 90,8\% refirió que le parecía adecuada la presencia de estudiantes, internos o becados en el box. El $95,8 \%$ de los pacientes refirió sentirse satisfecho con la atención recibida.

Conclusiones: Las cifras generales de satisfacción en la población estudiada son positivas. El cuestionario podría ser de utilidad para medir satisfacción usuaria. Es necesario seguir monitorizando la percepción de los pacientes sobre la atención médica.
\end{abstract}

Palabras clave: Satisfacción usuaria en salud, atención médica, educación médica.

\section{ABSTRACT}

Introduction: Patient satisfaction with respect to health care institutions is relevant.

Aim: To perform content validation a questionnaire that allows to analyze the opinion of a group of patients attending an ENT clinic regarding on the process of care in this place.

\footnotetext{
Médico, Servicio de Otorrinolaringología, Hospital Barros Luco Trudeau, Universidad de Chile.

2 Programa de Doctorado en Salud Pública, Universidad de Chile.

3 Médico Cirujano, Universidad de Chile.
}

Recibido el 11 de julio, 2013. Aceptado para publicación el 3 de agosto, 2014. 
Material and method: Cross sectional study. The instrument was validated through Lawshe method. Seven reagents were created, with a total of 83 questions to be subjected to analysis.

Results: We validated 25 questions that made the final questionnaire, with a content validity ratio of 0.75 or more. We surveyed 120 patients between 30 and 60 years, being 83 women (69.1\%) and 37 men (30.9\%). Of the total, 75 patients (62.5\%) came to first consultation, and 36 patients (30\%) had been operated. More than $94 \%$ of the patients said that the doctor clearly explained his diagnosis and clearly gave indications to treat their condition. $90.8 \%$ said that it seemed appropriate the presence of students, interns and residents or fellows in the consultation box. $95.8 \%$ of patients reported being satisfied with the care provided.

Conclusions: The overall high level of satisfaction in the study population is positive. The questionnaire could be useful for measuring user satisfaction. It is necessary to continue to monitor patients' perception of care.

Key words: User satisfaction in health care, healthcare, medical education.

\section{INTRODUCCIÓN}

Hoy en día es común evaluar la calidad de los centros de salud mediante la medición del nivel de satisfacción de sus usuarios ${ }^{1,2}$. Una de las formas más habituales de participación de los usuarios es mediante encuestas de satisfacción, las que permiten que los usuarios valoren tanto el servicio recibido como sus componentes ${ }^{3}$; sin embargo, los factores más influyentes al momento de determinar la satisfacción del paciente son los de tipo perceptivo ${ }^{4}$. De esta manera, se pueden introducir mejoras en la gestión de forma que se ofrezca un servicio que sea y se perciba de mayor calidad, lo que podría redundar en el incremento de la satisfacción de los pacientes $y$, por ende, en el mejoramiento de los servicios sanitarios y la atención de salud ${ }^{5}$. Todo lo anterior guiado por la premisa de que un paciente satisfecho se muestra más predispuesto a seguir las recomendaciones médicas y terapéuticas $y$, por tanto, a mejorar su salud 6 . Esto, se refleja en la consideración actual de la satisfacción usuaria como outcome o resultado deseable en la atención de salud 7 . De hecho, varios autores han reportado la alta asociación existente entre la satisfacción del usuario y la calidad del servicio, lo que implica la necesidad del conocimiento de la opinión del usuario para conocer y mejorar la atención del mismo ${ }^{8,9}$.

\section{OBJETIVO}

El objetivo del presente estudio fue realizar validación de contenido de un cuestionario diseñado para analizar la opinión de un grupo de pacientes consultantes al Policlínico de Otorrinolaringología del Hospital Barros Luco Trudeau respecto al proceso de atención médica.

\section{MATERIAL Y MÉTODO}

Estudio de corte transversal en pacientes consultantes ambulatorios del Policlínico de Otorrinolaringología del Hospital Barros Luco Trudeau. Para la presente validación, fueron incluidos pacientes mayores de 18 años, excluyendo pacientes alterados de conciencia, con facultades mentales perturbadas o incapaces de contestar el cuestionario. El estudio contó con aprobación del Comité de Ética del centro de estudio, y cada paciente fue debidamente informado de la naturaleza del cuestionario y firmó un consentimiento.

Como instrumento de recolección se utilizó un cuestionario con preguntas de selección múltiple, confeccionado especialmente para medir los niveles de satisfacción usuaria respecto a la atención médica. Previo a su aplicación, el contenido del cuestionario fue sometido a un proceso de validación tanto de apariencia como de contenido mediante cálculo de la "razón de validez de contenido" de Lawshe (CVR). Inicialmente se formuló un total de 83 preguntas agrupadas en 8 reactivos. El modelo de Lawshe ha demostrado ser de utilidad para dictaminar la calidad de un instrumento en función de la validez de contenido. Para la validación de este cuestionario en particular, se utilizó el modelo 
original de Lawshe con 9 jueces, dentro de los que se encontraban médicos otorrinolaringólogos, enfermeras de gestión del policlínico, académicos con experiencia en validación de instrumentos, y pacientes asistentes al policlínico. El criterio de aceptación original de Lawshe para 9 jueces era igual o superior a 0,75 en el CVR, lo que se cumplió para todos los reactivos que se incluyeron en la versión final del cuestionario. La versión final del cuestionario estuvo compuesta por 7 reactivos con 25 preguntas: "Atención médica", "Higiene en la atención", "Atención de paramédicos y personal no médico", "Atención de personal profesional no médico (enfermería y fonoaudiología)", "Resolución de problemas", "Tiempos de espera" y "Administrativo". De las 83 preguntas iniciales, 25 conformaron el cuestionario definitivo, todas ellas cumpliendo con el criterio mínimo de inclusión, es decir, una CVR $\geq 0,75$ (Lawshe, 1975).

Se estimó un tamaño muestral, mediante muestreo aleatorio simple, considerando $\alpha=0,05$ y una potencia estadística (1-ß) del 80\%, que arrojó 120 pacientes, para una diferencia de satisfacción de
$58 \%$ a nivel nacional y $70 \%$ a nivel local. Se utilizó el paquete estadístico Stata 12.1 (StataCorp. 2011, College Station, TX: StataCorp LP, EE.UU.)

\section{RESULTADOS}

Se validaron 25 preguntas que conformaron el cuestionario final, con una razón de validez de contenido de 0,75 o más. Fueron encuestados 120 pacientes entre 30 y 60 años, correspondientes a 83 mujeres $(69,1 \%)$ y 37 hombres $(30,9 \%)$. De los 120 pacientes, 75 pacientes (62,5\%) acudían por primera vez a consulta, y 36 pacientes (30\%) habían sido sometidos a una intervención quirúrgica.

Respecto al reactivo "Atención médica", más del $94 \%$ del total de pacientes refirió que el médico le explicó claramente su diagnóstico y le dio con claridad las indicaciones para tratar su enfermedad. El 90,83\% refirió que le parecía adecuada la presencia de estudiantes, internos o residentes en el box. El 95,83\% de los pacientes refirió sentirse satisfecho con la atención recibida (Tabla 1). Con

Tabla 1. Respuestas a cuestionario de satisfacción con la atención médica en un policlínico de otorrinolaringología

\begin{tabular}{|c|c|c|}
\hline REACTIVO: ATENCIÓN MÉDICA & & PORCENTAJE \\
\hline El médico me saludó cuando comenzó la consulta & $\begin{array}{l}\text { Insat. (\%) } \\
\text { Ind. }(\%) \\
\text { Sat. }(\%)\end{array}$ & $\begin{array}{l}2.5 \\
1.7 \\
95.8\end{array}$ \\
\hline Sentí que el médico me escuchaba atentamente mientras le contaba mi problema & $\begin{array}{l}\text { Insat. (\%) } \\
\text { Ind. }(\%) \\
\text { Sat. }(\%)\end{array}$ & $\begin{array}{l}3.3 \\
4.2 \\
92.5\end{array}$ \\
\hline El médico me explicó claramente mi diagnóstico & $\begin{array}{l}\text { Insat. (\%) } \\
\text { Ind. }(\%) \\
\text { Sat. }(\%)\end{array}$ & $\begin{array}{l}2.5 \\
3.4 \\
94.1\end{array}$ \\
\hline El médico me dio con claridad las indicaciones para tratar mi enfermedad & $\begin{array}{l}\text { Insat. (\%) } \\
\text { Ind. }(\%) \\
\text { Sat. }(\%)\end{array}$ & $\begin{array}{c}2.5 \\
5 \\
92.5\end{array}$ \\
\hline El médico tuvo un trato agradable y respetuoso durante la entrevista & $\begin{array}{l}\text { Insat. (\%) } \\
\text { Ind. }(\%) \\
\text { Sat. }(\%)\end{array}$ & $\begin{array}{c}1.6 \\
2.6 \\
95.8\end{array}$ \\
\hline El médico utilizó un lenguaje claro y adecuado mientras me atendió & $\begin{array}{l}\text { Insat. (\%) } \\
\text { Ind. }(\%) \\
\text { Sat. }(\%)\end{array}$ & $\begin{array}{c}3.3 \\
0.9 \\
95.8\end{array}$ \\
\hline Es adecuada la presencia de estudiantes, internos o becados de medicina & $\begin{array}{l}\text { Insat. (\%) } \\
\text { Ind. }(\%) \\
\text { Sat. }(\%)\end{array}$ & $\begin{array}{l}4.1 \\
5.1 \\
90.8\end{array}$ \\
\hline Me siento conforme con la atención recibida en general & $\begin{array}{l}\text { Insat. (\%) } \\
\text { Ind. }(\%) \\
\text { Sat. }(\%)\end{array}$ & $\begin{array}{c}3.3 \\
0.9 \\
95.8\end{array}$ \\
\hline
\end{tabular}

* Insat.: insatisfecho / Ind.: indiferente / Sat.: satisfecho 
respecto a la "Higiene en la atención", cuando se preguntó por la presentación personal del equipo de salud se obtuvo $96,6 \%$ de satisfacción. Cuando se preguntó por la higiene de los insumos utilizados en la atención se obtuvo 93,3\% de satisfacción. Finalmente, cuando se preguntó por la higiene del box de atención se obtuvo 96,6\% de satisfacción (Tabla 2). En relación al ítem "Resolución de problemas", se obtuvieron adecuados grados de satisfacción, como se detalla en la Tabla 2 y en la Figura 1.

En relación con la "Atención del personal no profesional (paramédico y administrativo)" y a la "Atención de profesional no médico (enfermería y fonoaudiología)" se obtuvieron resultados satisfactorios, que se detallan en la Tabla 3. Finalmente, en cuanto a la satisfacción con la atención expresada por los pacientes encuestados relacionado con los "tiempos de espera" y los "aspectos administrativos" de su atención, últimos ítems de la encuesta, se aprecia que la pregunta que obtuvo mayor satisfacción fue la relativa a la atención médica el día programado de su control $(94,2 \%)$, y la que obtuvo la menor satisfacción fue la referente a la posibilidad de cambio de su hora médica por algún problema (44,2\%) (Tabla 4).

\section{DISCUSIÓN}

En los últimos años, el estudio de la satisfacción usuaria respecto a los servicios de salud se ha convertido en un instrumento de valor creciente tanto para las instituciones como en la investigación en salud. Los sistemas de salud presentan, junto con las características propias del sector, otros aspectos, como intangibilidad, heterogeneidad, simultaneidad de la producción y consumo, lo que hace especialmente complejo su análisis ${ }^{10}$.

Las cifras generales de satisfacción en la población estudiada $(95,8 \%)$ son superiores a los referidos a nivel nacional. En 2001, Alvarado y cols ${ }^{11}$

Tabla 2. Respuestas a cuestionario de satisfacción respecto a higiene de la atención y a resolución de problemas en un policlínico de otorrinolaringología

\begin{tabular}{|l|c|c|}
\hline REACTIVO: HIGIENE DE LA ATENCIÓN & & PORCENTAJE \\
\hline Me pareció que los integrantes del equipo de salud que me atendieron tenían & Insat. (\%) & 1.6 \\
una buena presentación personal & Ind. (\%) & 1.8 \\
& Sat. (\%) & 96.6 \\
\hline El médico utilizó material limpio y sellado para limpiarme y curarme en caso & Insat. (\%) & 1.6 \\
que haya tenido alguna herida o que me haya hecho algún aseo. & Ind. (\%) & 5,1 \\
& Sat. (\%) & 93.3 \\
\hline El box de atención se veía limpio y ordenado & Insat. (\%) & 1.6 \\
& Ind. (\%) & 1.8 \\
\hline REACTIVO: RESOLUCION DE PROBLEMAS & Sat. (\%) & 96.6 \\
\hline Una vez con mi diagnóstico comencé rápidamente con el tratamiento & \multicolumn{2}{|c|}{} \\
& Insat. (\%) & 2.5 \\
\hline Comprendí la dosis de los fármacos administrados & Ind. (\%) & 10.8 \\
& Sat. (\%) & 86.7 \\
\hline Se explicó cada procedimiento que se me realizó & Insat. (\%) & 0.8 \\
& Ind. (\%) & 17.5 \\
\hline Recibí de forma clara información sobre mi patología y posterior tratamiento & Sat. (\%) & 81.7 \\
\hline
\end{tabular}

* Insat.: insatisfecho / Ind.: indiferente / Sat.: satisfecho 


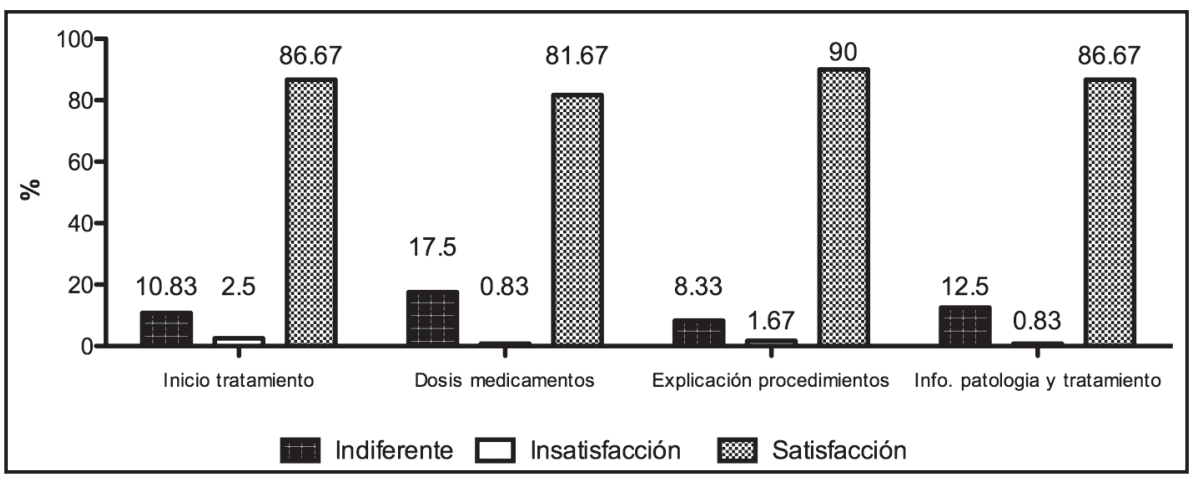

Figura 1. Distribución porcentual de respuestas a cuestionario de satisfacción de la atención de salud en relación a la resolución de problemas.

Tabla 3. Respuestas a cuestionario de satisfacción con la atención del personal no profesional (paramédico y administrativo) y profesional no médico (enfermería y fonoaudiología) en un policlínico de otorrinolaringología

\begin{tabular}{|c|c|c|}
\hline REACTIVO: ATENCIÓN PERSONAL NO PROFESIONAL & & PORCENTAJE \\
\hline $\begin{array}{l}\text { Los técnicos que me atendieron tuvieron un trato respetuoso y cuidadoso conmigo } \\
\text { durante la sesión }\end{array}$ & $\begin{array}{l}\text { Insat. (\%) } \\
\text { Ind. (\%) } \\
\text { Sat. (\%) }\end{array}$ & $\begin{array}{c}1.7 \\
4.1 \\
94.2\end{array}$ \\
\hline El personal administrativo (ventanilla) fue respetuoso y amable en su trato & $\begin{array}{l}\text { Insat. (\%) } \\
\text { Ind. }(\%) \\
\text { Sat. }(\%)\end{array}$ & $\begin{array}{c}10.8 \\
5 \\
84.2\end{array}$ \\
\hline Los técnicos fueron cuidadosos cuando me realizaron algún procedimiento durante mi atención & $\begin{array}{l}\text { Insat. (\%) } \\
\text { Ind. (\%) } \\
\text { Sat. (\%) }\end{array}$ & $\begin{array}{c}1.7 \\
8 \\
90.3\end{array}$ \\
\hline \multicolumn{3}{|l|}{ REACTIVO: ATENCION PROFESIONALES NO MÉDICOS (ENFERMERÍA Y FONOAUDIOLOGÍA) } \\
\hline Recibí un saludo respetuoso por parte de estos profesionales & $\begin{array}{l}\text { Insat. (\%) } \\
\text { Ind. (\%) } \\
\text { Sat. (\%) }\end{array}$ & $\begin{array}{c}0 \\
15.8 \\
84.2\end{array}$ \\
\hline ¿Responden las preguntas que realizo? & $\begin{array}{l}\text { Insat. (\%) } \\
\text { Ind. (\%) } \\
\text { Sat. (\%) }\end{array}$ & $\begin{array}{c}0 \\
19.2 \\
80.8\end{array}$ \\
\hline Obtuve información con un lenguaje claro y asequible & $\begin{array}{l}\text { Insat. (\%) } \\
\text { Ind. (\%) } \\
\text { Sat. (\%) }\end{array}$ & $\begin{array}{c}1.7 \\
15 \\
83.3\end{array}$ \\
\hline
\end{tabular}

* Insat.: insatisfecho / Ind.: indiferente / Sat.: satisfecho

reportaron que la mayor parte de los encuestados evaluaban en forma satisfactoria la atención que recibían, pues el $87,7 \%$ de los pacientes hospitalizados, $69,4 \%$ de policlínico y $65 \%$ de los servicios de urgencia, calificaron la atención como buena 0 muy buena, en hospital público del país. Por otro lado, en 2012, Lobos y cols ${ }^{12}$ reportan $92,9 \%$ y
91,4\% de satisfacción general con la calidad de la atención en hospitales públicos docentes y no docentes, respectivamente.

Las variables aquí estudiadas que concitan el mayor grado de satisfacción son aquellas relacionadas con la atención médica, en especial las relativas al trato respetuoso, lenguaje adecuado y 
Tabla 4. Respuestas a cuestionario de satisfacción en relación a los tiempos de espera y aspectos administrativos en un policlínico de otorrinolaringología

\begin{tabular}{|c|c|c|}
\hline \multicolumn{2}{|l|}{ REACTIVO: TIEMPOS DE ESPERA } & PORCENTAJE \\
\hline Me atendieron el día de mi citación a control & $\begin{array}{r}\text { Insat. }(\%) \\
\text { Ind. }(\%) \\
\text { Sat. }(\%)\end{array}$ & $\begin{array}{c}3,3 \\
2,5 \\
94,2\end{array}$ \\
\hline El profesional de salud se tomo el tiempo suficiente para aclarar mis dudas & $\begin{array}{r}\text { Insat. }(\%) \\
\text { Ind. }(\%) \\
\text { Sat. }(\%)\end{array}$ & $\begin{array}{c}2,5 \\
4,2 \\
93,3\end{array}$ \\
\hline \multicolumn{3}{|l|}{ REACTIVO: ADMINISTRATIVO } \\
\hline Obtuve información sobre el día del resultado de mis exámenes & $\begin{array}{r}\text { Insat. }(\%) \\
\text { Ind. }(\%) \\
\text { Sat. }(\%)\end{array}$ & $\begin{array}{c}0,8 \\
15 \\
84,2\end{array}$ \\
\hline Pude cambiar mi hora médica por problemas personales u otros & $\begin{array}{r}\text { Insat. }(\%) \\
\text { Ind. }(\%) \\
\text { Sat. }(\%)\end{array}$ & $\begin{array}{c}1,7 \\
54,1 \\
442\end{array}$ \\
\hline
\end{tabular}

* Insat.: insatisfecho / Ind.: indiferente / Sat.: satisfecho

visión general de la atención del médico. Por otro lado, las variables que aglutinan el mayor grado de insatisfacción son aquellas relacionadas a la percepción de resolución de problemas, en particular aspectos relativos a comprender el tratamiento indicado, la rapidez de inicio del tratamiento, y la información sobre la patología. Este aspecto debe llamar la atención de los encargados de gestión de la atención, de manera tal de poder mejorar estos indicadores. Otro aspecto que mostró menor satisfacción fue el trato del personal administrativo hacia los pacientes. Algo similar se ha visto en estudios previos, que muestran que la característica que proporciona una mayor influencia por sobre la satisfacción del paciente, es la relación interpersonal con el personal no médico y los aspectos relacionados con la atención y comunicación del paciente con los médicos ${ }^{4}$.

La actual ley de deberes y derechos de las personas en salud enfatiza la importancia del tema aquí expuesto, pues se resguarda el derecho de todos los pacientes de recibir un trato digno, tener compañía y asistencia espiritual, efectuar consultas y reclamos, ser informados y consentir con conocimiento informado, por lo que los equipos de salud deben enfocar sus esfuerzos en satisfacer estos derechos.
En otorrinolaringología existen varios reportes de estimaciones de la satisfacción usuaria. Entre las más llamativas existe la de Schmidt y $\operatorname{cols}^{13}$ donde los pacientes consultantes de ORL destacaban positivamente la calidad de la atención médica y negativamente, aspectos relativos al tiempo de espera e infraestructura. Por otro lado existe el estudio de Boss y cols $^{14}$, que muestra adecuada satisfacción con los servicios generales en instituciones docentes y no docentes, con menor satisfacción en aspectos de la comunicación entre el equipo de salud y pacientes. Finalmente, se destaca la experiencia en satisfacción usuaria en otorrinolaringología pediátrica de Zopf y cols ${ }^{15}$, que mostraron excelente aprobación de la atención de salud (cercano al 100\% de satisfacción), con ciertos reparos en la infraestructura y tiempos de espera.

El presente estudio incluyó una pregunta en relación a la presencia de estudiantes en la atención de salud, obteniendo 90,8\% de satisfacción con respecto a ella, lo que se debe a la percepción general que tiene el paciente en cuanto a participar en el proceso de aprendizaje y que se sienten más comprendidos y escuchados en la presencia de estudiantes ${ }^{12}$. No ocurre lo mismo en otros países donde los pacientes tienden a ver con menor satis- 
facción la participación de la docencia en la atención de salud pues algunos lo consideran como un factor que enlentece la atención ${ }^{16}$. En cualquier caso, esto respalda la presencia de procesos de formación médica en hospitales docentes como algo positivo.

En conclusión, consideramos que es necesario seguir monitorizando la percepción de los pacientes sobre la atención médica, para mejorar aquellos aspectos deficitarios con el fin de hacer más satisfactoria la experiencia de aquellos que sufren una enfermedad, y eventualmente optimizar los procesos médicos.

Este estudio no contó con apoyo financiero de ninguna institución.

\section{BIBLIOGRAFÍA}

1. Ruiz M, Martínez G, Calvo J, Aguirre h, Arango $R$, LaRA R, et AL. Bases para la evaluación de la calidad de la atención en las unidades médicas del sector salud. Salud Pública Mex 1990; 32: 156-69.

2. Clearly PD, Edgman-Levitan S, Roberts M, Moloney TW, McMullen W, Walker JD, et al. Patients evaluate their hospital care: A national survey. Health Affairs 1991; 10: 254-67.

3. Serrano-del Rosal R, Biedma L. El usuario del sistema sanitario: gestor de calidad. IX Congreso de Metodología de las Ciencias Sociales y de la Salud. Granada: Libro de resúmenes, 2005; 262.

4. Castillo L, Dougnac A, Vicente I, Munoz V, Rojas V. Los predictores de satisfacción de pacientes en un centro hospitalario universitario. Rev Méd Chile 2007; 135(6): 696-701.

5. FitzPatrick R. Surveys of patient satisfaction: Important general considerations. $\mathrm{Br}$ Med $\mathrm{J}$ 1991; 302: 887-9.
6. Mira JJ, Vitaller J, Aranaz J, Herrero Jf, Buil JA. La satisfacción del paciente. Concepto y aspectos metodológicos. Revista de Psicología de la Salud 1992; 4: 89-116.

7. Verbeek J. Patient satisfaction: is it a measure for the outcome of care or the process of care? $J$ Clin Epidemiol 2004; 57(2): 217.

8. Mabry RL. Patient healing vs customer satisfaction. Otolaryngol Head Neck Surg 2006; 134(4): 715.

9. Kinney WC. A simple and valuable approach for measuring customer satisfaction. Otolaryngol Head Neck Surg 2005; 133(2): 169-72.

10. Parra P, Bermejo R, Más A, Hidalgo M, Gomis R, Calle J. Factores relacionados con la satisfacción del paciente en los servicios de urgencia hospitalarios. Gac Sanit 2012; 26: 159-65.

11. Alvarado R, Vera A. Evaluación de la satisfacción de usuarios de hospitales del sistema nacional de servicios de salud en Chile. Rev Chilena Salud Pública 2001; 5(2/3): 81-9.

12. Lobos A, Cardemil F, loézar C, González V, Ortega A. Satisfacción con la atención médica de pacientes hospitalizados en un servicio clínico docente y en uno no docente. Rev Chil Salud Pública 2012; Vol 16(1): 16-25.

13. Schmidt K, Meyer J, Jahnke I, Wollenberg B, SCHMIDT C. Patient satisfaction in the outpatient department: a pilot study for customer satisfaction in ENT. HNO 2009; 57(3): 251-6.

14. Boss EF, Thompson RE. Patient satisfaction in otolaryngology: Can academic institutions compete? Laryngoscope 2012; 122(5): 10009.

15. Zopf D, Joseph AW, Thorne MC. Patient and family satisfaction in a pediatric otolaryngology clinic. Int J Pediatr Otorhinolaryngol 2012; 76(9): 133942.

16. Boss EF, Thompson RE. Patient experience in the pediatric otolaryngology clinic: does the teaching setting influence parent satisfaction? Int J Pediatr Otorhinolaryngol 2013; 77(1): 59-64. 